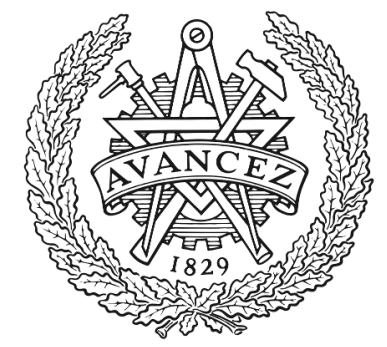

CHALMERS

UNIVERSITY OF TECHNOLOGY

\title{
Energy Scheduling Strategies for Grid-connected Microgrids: A Case Study on Chalmers Campus
}

Downloaded from: https://research.chalmers.se, 2023-04-26 06:02 UTC

Citation for the original published paper (version of record):

Antoniadou-Plytaria, K., Steen, D., Le, A. et al (2019). Energy Scheduling Strategies for Grid-connected Microgrids: A Case Study on Chalmers Campus. Proceedings of 2019 IEEE PES Innovative Smart Grid Technologies Europe, ISGT-Europe 2019.

http://dx.doi.org/10.1109/ISGTEurope.2019.8905472

N.B. When citing this work, cite the original published paper. 


\title{
Energy Scheduling Strategies for Grid-connected Microgrids: A Case Study on Chalmers Campus
}

\author{
Kyriaki Antoniadou-Plytaria, David Steen, Le Anh Tuan and Ola Carlson \\ Department of Electrical Engineering \\ Chalmers University of Technology \\ 41296 Gothenburg, Sweden \\ Email: \{kyriaki.antoniadou, david.steen, tuan.le, ola.carlson\}@chalmers.se
}

\begin{abstract}
This paper focuses on the optimal energy management of grid-connected microgrids with battery energy storage systems. The microgrid energy management and the optimal power flow of the distribution network are formulated as mixedinteger linear optimization problems to evaluate microgrid energy scheduling strategies including cost minimization, maximum use of own resources, and minimum energy exchange with the upstream network. The real distribution network of Chalmers University of Technology campus is used as a case study. The study results show that economic optimization yields an annual microgrid cost reduction of $4 \%$. Alternatively, if the microgrid minimizes the energy exchange, virtual islanding operation (zero energy exchange) for 3211 hours can be achieved within a year. The results also present the effects on the operation and cost of the distribution system and highlight a trade-off between microgrid cost minimization and battery lifetime.

Index Terms-Battery energy storage system, distribution system operator, energy management system, energy scheduling, grid-connected microgrids, microgrids, optimization.
\end{abstract}

\section{INTRODUCTION}

The need to have uninterrupted and reliable power supply in remote locations or areas has so far been the driving force for the deployment of microgrids (MGs) [1] around the globe with the main focus being in islanding capability and grid-forming control. The key component of the MG is the MG controller, which manages the producing and consuming units (distributed generation, flexible loads, storage) that are clustered together to form the MG. Part of the MG controller is the energy management system (EMS), which is found at the tertiary level of hierarchical MG control [2]. The task of the EMS is to optimally balance load and supply both in the planning phase and in the delivery phase. Therefore, the adoption of MGs can address the volatility in intermittent renewable-based generation and plug-in electric vehicles (PEV) charging and enhance the utilization of fossil-free energy sources.

In Europe, the development of MGs has not been significantly promoted yet, which is evident by the lack of regulations and policies on this concept [3]. However, with the latest decrease in the battery cost, the installation of behindthe-meter energy storage can be combined with residential

This work has received funding in the framework of the joint programming initiative ERANet Smart Energy Systems focus initiative Smart Grids Plus, with support from the European Unions Horizon 2020 research and innovation programme under grant agreement No 646039. photovoltaics (PVs) to increase the self-supply level of endusers during the day. This will increase the share of distributed energy resources (DERs) at the distribution level and could give rise to grid-connected MGs. Therefore, the future paradigm of distribution management system (DMS) integrates the microgrid energy management system (MG-EMS).

Recent research has studied the optimal energy scheduling for MGs with battery energy storage systems (BESS) for MG-EMS that operate in uncoordinated schemes [4], [5] and for the coordinated operation of multiple MG-EMS assuming interaction with the distribution system [6]-[8]. The modeling of the distribution network is often ignored or simplified using DC optimal power flow (DC-OPF). In addition, most of these studies do not consider BESS degradation or if it is included [4], the long-term effects are not evaluated. All these works assume that both the DSO and the MGs can own and schedule DER and they trade energy with each other.

This work considers the unbundled structure instead, in which the DSO does not own DER and no energy trading is considered between the DSO and the MGs. The MGs are separate entities operated by a different stakeholder and they can contribute to the management of the distribution network by modifying the dispatch of their resources to control the power exchange at the physical interface with the network, i.e. the point of common coupling (PCC).

The aim of this paper is to evaluate optimal energy scheduling strategies of grid-connected MGs with BESS and identify benefits and challenges regarding the operation and cost of the MGs and the distribution system. The MG-EMS integration to the DMS is a part of the m2M-GRID project [9] that studies microgrid interactions in active distribution networks.

The contributions of this paper are threefold:

- It describes and compares multi-period optimization models for energy scheduling of MG resources both in uncoordinated and in coordinated schemes, where AC OPF is used for the distribution network modeling.

- It presents a case study on the real distribution network of a university campus, which has been performed for a year considering various operational strategies. The reallife environment of the campus adds credibility to such case studies [10], [11].

- It provides a link between the applied energy scheduling strategy and the expected battery lifetime, which is cal- 
culated based on the rainflow cycle-based counting. To the best of the authors' knowledge this link has not been presented in any of the previous literature.

The remaining of the paper is organized as follows: Section II provides the mathematical formulation and Section III presents the case study and the operational strategies. The results are discussed in Section IV and the conclusions are drawn in Section V.

\section{Mathematical Formulation}

\section{A. Optimal Energy Scheduling for a Grid-connected MG}

The MG operators seek to optimally schedule the available DER, while satisfying power balance within the MG and the operational constraints of the resources. The DER considered in this work include: dispatchable distributed generation provided by a bio-fuel based combined heat and power (CHP) plant, non-dispatchable distributed generation (DG) of PVs, distributed BESS and demand response resources (DRR).

1) Objective Functions: The optimal energy scheduling problem presented in this section is defined for two different objective functions: the profit maximization and the energy exchange minimization. These objective functions are generic in their formulation, as they consider both imported and exported energy, and thus they can be used with different MGEMS strategies, which can be related e.g., with cost, income or level of interaction (energy exchange) with the main grid.

The objective function for profit maximization is

$$
\begin{array}{r}
\max \sum_{t \in \mathcal{T}}\left(c^{s p o t}+c_{e}\right) P_{i, t}^{e x}-\left(c^{s p o t}+c_{i}\right) P_{i, t}^{i m} \\
-c^{C H P} P_{t}^{H}-R_{i}^{p}, \forall i \in \mathcal{M G}
\end{array}
$$

where the first term is the income from selling energy and the other terms correspond to the total cost, which consists of the imported energy cost, the generation cost, as well as the network charges that include energy transmission (grid utilization) cost and peak imported power cost. These charges are paid to the distribution system operator (DSO).

In (1), $\mathcal{M G}$ is the set of the MGs' PCC with the distribution network and $\mathcal{T}$ is the simulation horizon, which is 24 hours with hourly time steps (therefore the time step is omitted from the mathematical formulation). The positive variables $P_{i, t}^{i m} / P_{i, t}^{e x}$ denote the MG imported/exported power from/to the upstream network through the PCC at bus $i$. The heating output of the CHP is treated as a parameter and is denoted by $P_{t}^{H}$. Moreover, $c^{C H P}$ refers to the fuel cost of the CHP, $c^{\text {spot }}$ is the spot price, $c_{i}$ is the grid charge for energy transmission, when the MG imports energy, $c_{e}$ is the reimbursement paid to the MG by the DSO (as an incentive to reduce network losses), when the MG exports energy, and $R_{i}^{p}$ is the cost for the peak power drawn from the main grid (measured on an hourly basis), which must be constrained by

$$
R_{i}^{p} \geq C_{p p}^{M G} P_{i, t}^{i m}, \forall i \in \mathcal{M G}, t \in \mathcal{T}
$$

where $C_{p p}^{M G}$ is the power-based grid tariff.

The objective function for the case of energy exchange minimization is:

$$
\min \sum_{t \in \mathcal{T}} P_{i, t}^{e x}+P_{i, t}^{i m}, \forall i \in \mathcal{M G}
$$

2) $M G$ Energy Balance: The active and reactive power balance of the MG are given by

$$
\begin{gathered}
\sum_{j \in \mathcal{N}}\left(P_{j, t}^{G}+P_{j, t}^{P V}+P_{j, t}^{D S,-}+P_{j, t}^{D R}-P_{j, t}^{L}-P_{j, t}^{D S,+}\right) \\
+P_{i, t}^{i m}-P_{i, t}^{e x}=0 \\
\sum_{j \in \mathcal{N}}\left(Q_{j, t}^{G}+Q_{j, t}^{D R}-Q_{j, t}^{L}\right)+Q_{i, t}^{i m}=0
\end{gathered}
$$

for all $i \in \mathcal{M G}, t \in \mathcal{T}$, where $\mathcal{N}$ denotes the set of MG buses (including the PCC bus). Constraint (4) defines the active power balance, where $P_{j, t}^{G}, P_{j, t}^{P V}, P_{j, t}^{L}, P_{j, t}^{D S,+} / P_{j, t}^{D S,-}$, and $P_{i, t}^{D R}$ refer to dispatchable generation (CHP), non-dispatchable generation (PV), load, charging/discharging power from distributed BESS and curtailed (or increased) power from DRR, respectively. Similarly, constraint (5) defines the reactive power balance, where constant power factor is considered for generation and load (flexible and inflexible).

3) Battery Energy Storage System: The BESS must satisfy

$$
\begin{gathered}
S O C_{j, t}=S O C_{j, t-1}+\eta \frac{P_{j, t}^{D S,+}}{E_{\max }}-\frac{P_{j, t}^{D S,-}}{\eta E_{\max }} \\
S O C_{j, \min } \leq S O C_{j, t} \leq S O C_{j, \max } \\
0 \leq P_{j, t}^{D S,+} \leq \kappa_{j}^{D S} E_{\max }, 0 \leq P_{j, t}^{D S,-} \leq \kappa_{j}^{D S} E_{\max } \\
P_{j, t}^{D S,+} \leq z_{j, t}^{+} M, P_{j, t}^{D S,+} \leq z_{j, t}^{-} M, z_{j, t}^{+}+z_{j, t}^{-} \leq 1
\end{gathered}
$$

for all $j \in \mathcal{N}, t \in \mathcal{T}$. The power to energy ratio, which depends on the energy storage technology and limits the maximum charging or discharging power is denoted by $\kappa_{j}^{D S}$, while $\eta$ is the charging/discharging efficiency (assumed to be the same), $E_{\max }$ is the installed capacity and $S O C_{j, t}$ is the stateof-charge (SOC), which must lie between the lower and upper limit $\left(S O C_{j, \min }\right.$ and $\left.S O C_{j, \max }\right)$, respectively. The binary variables $z_{j, t}^{+} / z_{j, t}^{-}$indicate if the BESS is charging/discharging and $M$ is a very large number.

4) Demand Response (DR): The model for load flexibility is equivalent to the energy storage model and is described by the constraints on the energy that is available for DR:

$$
\begin{gathered}
E_{j, t}^{D R}=E_{j, t-1}^{D R}+P_{j, t}^{D R} \\
0 \leq E_{j, t}^{D R} \leq \nu P_{j, t}^{L} \\
-\kappa_{j}^{D R} \nu P_{j, t}^{L} \leq P_{j, t}^{D R} \leq \kappa_{j}^{D R} \nu P_{j, t}^{L}
\end{gathered}
$$

for all $j \in \mathcal{N}, t \in \mathcal{T}$. In these formulas, $E_{j, t}^{D R}$ is the energy that has already been curtailed at time $t$. The value of the maximum energy capacity of DRR is given as a percentage $\nu$ of the load $P_{j, t}^{L}$ at MG bus $j$ and $\kappa_{j}^{D R}$ is the power to energy ratio, which depends on the technology of the DRR [12]. 
5) CHP Plant: The electric output of the CHP plant is constrained by

$$
P_{\text {min }}^{G} \leq P_{j, t}^{G} \leq P_{t}^{H} r^{C H P}, \forall j \in \mathcal{N}, t \in \mathcal{T}
$$

where $P_{\text {min }}^{G}$ is the minimum electric power output and $r^{C H P}$ is the ratio of electric power to heating power output, which depends on the type of fuel and the operating point of the electric generator.

The formulation of the optimal scheduling problem for the MG profit maximization is given by (1)-(2) and (4)-(13), while the optimal scheduling problem for the minimization of the MG's energy exchange is (3)-(13). Both models define mixed-integer linear programming (MILP) problems. Since no interaction is considered with the DSO, these models solve the uncoordinated MG energy scheduling problem.

\section{B. AC OPF for the Distribution Network}

The coordinated energy scheduling model presented here is formulated as an AC OPF problem, since the purpose is to find the energy scheduling that would optimize the operation of the distribution network, if the MGs would empower the DSO to dispatch the DER.

1) Objective function: The DSO can achieve economic operation by minimizing the charges (energy transmission and peak power charges) paid to the upstream network (the transmission system operator):

$$
\min \sum_{t \in \mathcal{T}} P_{i, t}^{S S} c_{i}+R_{i}^{S S, p}, \forall i \in \mathcal{D}_{s}, t \in \mathcal{T}
$$

In (14), $\mathcal{D}_{s} \subseteq \mathcal{D}$ is the subset of substation buses (connection to the upstream network), which belong to the set of distribution network buses $\mathcal{D}$ (which also includes the MG buses), $P_{i, t}^{S S}$ is the active power at the substation, and $c_{i}$ is the energy transmission charge. The cost of the peak power measured at the substation is denoted by $R^{S S, p}$, which is constrained by

$$
R_{i}^{S S, p} \geq C_{p p}^{D S O} P_{i, t}^{S S}, \forall i \in \mathcal{D}_{s}, t \in \mathcal{T}
$$

where $C_{p p}^{D S O}$ is the power-based grid tariff.

2) Power Flow Constraints: The linearized Branch Flow Model [13], where the power losses are assumed to be negligible, is used for the network power flow:

$$
\begin{aligned}
P_{j, t}^{S S}+P_{j, t}^{G}+P_{j, t}^{P V}+P_{j, t}^{D R}+ & P_{j, t}^{D S,-}-P_{j, t}^{L}-P_{j, t}^{D S,+} \\
& =\sum_{i: j \sim i} P_{j i, t}-\sum_{i: i \sim j} P_{i j, t} \\
Q_{j, t}^{S S}+Q_{j, t}^{G}+Q_{j, t}^{D R}-Q_{j, t}^{L} & =\sum_{i: j \sim i} Q_{j i, t}-\sum_{i: i \sim j} Q_{i j, t}
\end{aligned}
$$

for all $j \in \mathcal{D}, t \in \mathcal{T}$ as well as

$$
\begin{array}{r}
v_{j, t}=v_{i, t}-2\left(R_{i j} P_{i j, t}+X_{i j} Q_{i j, t}\right) \\
\forall(i, j): i \sim j, \forall i, j \in \mathcal{D}, t \in \mathcal{T}
\end{array}
$$

where $v_{i, t}=\left|V_{i}, t\right|^{2}\left(\left|V_{i}, t\right|\right.$ is the voltage magnitude).

3) Voltage Limits: Voltage must lie within statutory limits:

$$
V_{\text {min }} \leq\left|V_{i}, t\right| \leq V_{\text {max }}, \forall i \in \mathcal{D}, t \in \mathcal{T}
$$

The coordinated MG energy scheduling problem is defined by (14), (15), (16)-(19), and (6)-(13) and is a MILP problem.

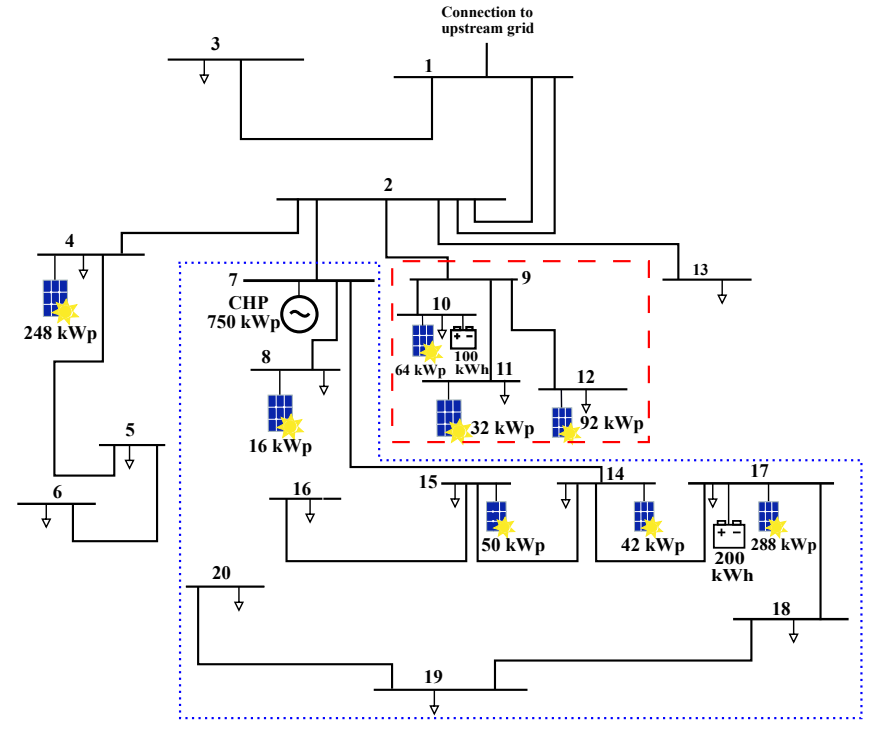

Fig. 1. The distribution network of Chalmers and the interconnection layout of the two MGs (shown with the sections in dashed and dotted lines).

\section{Case Study and Simulation Setups}

The electrical distribution network of Chalmers [10], which has a peak load of about $6 \mathrm{MW}$, was used to evaluate EMS strategies of grid-connected MGs (Fig. 1). The CHP boilers are primarily scheduled for heating energy production and the CHP plant is out of operation between April-September.

The assumptions of this case study are that the CHP plant has a fixed electric power output efficiency $\left(r^{C H P}=0.25\right)$ and operates under a constant leading power factor of 0.96 , while the load (both flexible and inflexible) has a constant lagging power factor of 0.98. Moreover, $\kappa_{j}^{D S}=0.5, \kappa_{j}^{D R}=0.5$, and $\nu=20 \%$. The SOC ranges between $20 \%$ to $90 \%$ and $\eta=0.95$, a value typically chosen for these studies [6].

Two network areas have been considered as MGs. Microgrid A (MGA), shown with dotted lines in Fig. 1, can produce excess energy, when the CHP is operating and it has a PV penetration level of $23 \%$ (as a percentage of the MG's peak load). Microgrid B (MGB), shown with the dashed lines in Fig. 1 only has PVs (5\%) as local generation and must continuously import from the main grid. The MGs are the only areas, where flexible load is available.

The case study uses the network data, resource topology and capacity data as well as historical data of electricity price, electricity and heating demand, temperature, and irradiation for 358 days of year 2016. The day-ahead energy scheduling problem is solved repeatedly for each day. The load data were acquired from smart meters at campus' buildings and were aggregated to be used as input to the OPF of the network. The PV generation was calculated according to [14]. The load and PV generation data are treated as "perfect forecasts".

\section{A. Energy Price and Grid Charges}

The Nord Pool spot market price [15] is used for the energy trade of the MGs, when they purchase or sell electricity. Moreover, when the MG operator sells generated electricity, 
TABLE I

PEAK IMPorted POWER AND ENERgy TRANSMission Cost

\begin{tabular}{ll}
\hline \hline \multicolumn{2}{c}{ MG company } \\
\hline Energy transmission cost & $68 \mathrm{SEK} / \mathrm{MWh}$ \\
Peak power tariff & 44 SEK/kW/month \\
\hline \hline \multicolumn{1}{c}{$\mathbf{1 0} \mathbf{~ k V}$ distribution grid } \\
\hline Energy transmission cost & $31 \mathrm{SEK} / \mathrm{MWh}$ \\
Peak power tariff & $37.1 \mathrm{SEK} / \mathrm{kW} /$ month \\
\hline \hline
\end{tabular}

TABLE II

ENERGY SCHEDULING STRATEGIES

\begin{tabular}{lccc}
\hline \hline & MGA & MGB & DSO \\
\hline Strategy 0 (BAU) & no EMS & no EMS & - \\
Strategy 1 (S1) & max. profit & min. cost & - \\
Strategy 2 (S2) & min. energy exchange & min. cost & - \\
Strategy 3 (S3) & min. import & min. import & - \\
Strategy 4 (S4) & - & - & min. cost \\
\hline \hline
\end{tabular}

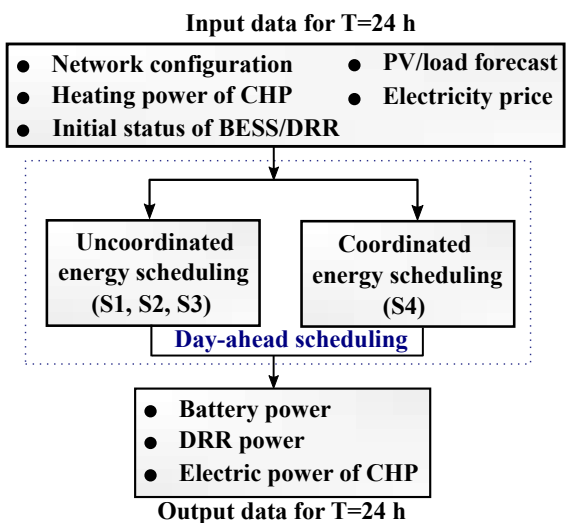

Fig. 2. The solution approach of the energy scheduling strategies.

a reimbursement of $30 \mathrm{SEK} / \mathrm{MWh}$ is received from the connected DSO [16]. The energy and power grid tariffs that have been used can be seen in Table I [16].

\section{B. Strategies}

The energy scheduling strategies are summarized in Table II. Strategy 0 is the business as usual (BAU) scenario, where the dispatch of the BESS follows a rule-based algorithm that triggers charging and discharging based on peak and low load thresholds. No DR is considered in BAU. Strategies 1-3 (S1S3) use the uncoordinated energy scheduling model, which is a local optimization model, and as it can be seen, the MGs might even have different objectives. Coordinated energy scheduling (global optimization) is considered in Strategy 4 (S4). The solution approach can be seen in Fig. 2.

\section{RESUlTS AND Discussions}

The optimization models were developed in GAMS [17] and the Cplex solver was used for the MILP problems. The metrics associated with the MGs and the main grid operation and cost are presented in Table III. The generation cost of the CHP is not included in the results because the boilers are primarily dispatched to supply heating demand and the additional cost for co-generating electricity is considered negligible.
TABLE III

Annual Cost and Performance Metrics

\begin{tabular}{lccccc}
\hline \hline \multicolumn{7}{c}{ BAU } & S1 & S2 & S3 & S4 \\
\hline \hline \multicolumn{7}{c}{ MGA } \\
\hline Annual cost (MSEK) & 0.90 & 0.86 & 0.96 & 0.91 & 0.90 \\
Imported energy (GWh) & 2.99 & 2.95 & 2.95 & 2.95 & 2.99 \\
Exported energy (MWh) & 337 & 296 & 0 & 295 & 329 \\
Zero exchange hours & 0 & 601 & 3211 & 725 & 0 \\
\hline \hline \multicolumn{7}{c}{ MGB } \\
\hline Annual cost (MSEK) & 2.11 & 2.08 & 2.08 & 2.14 & 2.11 \\
Imported energy (GWh) & 6.95 & 6.95 & 6.95 & 6.95 & 6.95 \\
\hline \hline \multicolumn{7}{c}{ DSO } \\
\hline Annual cost (MSEK) & 2.71 & 2.66 & 2.70 & 2.73 & 2.64 \\
Imported energy (GWh) & 28.72 & 28.72 & 29.02 & 28.72 & 28.72 \\
Peak power (MW) & 5.66 & 5.47 & 5.61 & 5.70 & 5.44 \\
\hline \hline
\end{tabular}
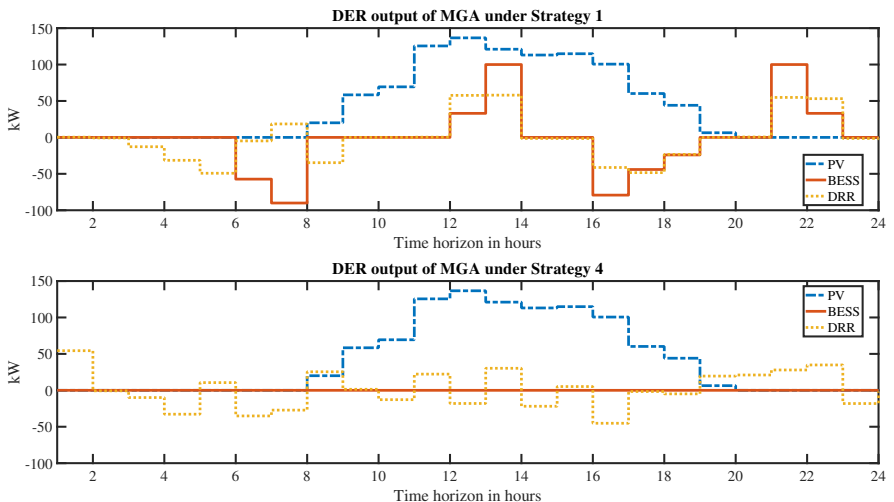

Fig. 3. The DER output of $M G A$ for an autumn day (comparison between S1 and S4).

\section{A. Cost of MGs and the DSO}

It can be seen that $\mathrm{S} 1$ reduces the cost of all three entities (MGA, MGB, DSO), compared to BAU, which means that all interconnected systems benefit from the deployment of two MG-EMS that seek to locally optimize the MG costs. The cost reduction is $2 \%$ for $M G B$ and DSO and $4 \%$ for $M G A$. If $\mathrm{S} 2$ is applied, the cost of $M G A$ is increased by $7 \%$ compared to BAU; however, the self-supply level of the MG is significantly improved. Specifically, the MG can operate as a virtual island and supply its customers with its own resources for 3211 hours (about 4.5 months) throughout the year.

The coordinated energy scheduling (S4), which yields the most economic operation for the DSO, results in costs similar or slightly reduced compared to BAU for both MGs; however, it is not the optimal solution for the MGs. As an example, Fig. 3 shows the variation in the BESS and DRR scheduling pattern of $M G A$ (positive values indicate discharging and load curtailment), when S1 and S4 are applied during an autumn day (the CHP is out of operation). In S4, the BESS will only be scheduled to contribute to the system's peak reduction (in Fig. 3 the batteries are not used for this day). Unlike S4, the scheduling in $\mathrm{S} 1$ is affected by the spot price and follows its fluctuation e.g., the BESS is discharged and load is curtailed from 13:00-14:00 at high PV production because the spot price has a high value at that hour.

The peak load consumption of the whole distribution system 
TABLE IV

BATTERY UTILIZATION

\begin{tabular}{cccccc}
\hline \hline \multicolumn{7}{c}{ BAU } & S1 & S2 & S3 & S4 \\
\hline \hline MGA \\
\hline Battery cycles & 67 & 403 & 153 & 144 & 317 \\
Average DoD \% & 78.5 & 80 & 80 & 80 & 80 \\
Lifetime in years & $>15$ & 11 & $>15$ & $>15$ & 14 \\
\hline \hline \multicolumn{7}{c}{ MGB } \\
\hline Battery cycles & 200 & 400 & 400 & 0 & 317 \\
Average DoD \% & 79 & 80 & 80 & - & 80 \\
Lifetime in years & $>15$ & 11 & 11 & - & 14 \\
\hline \hline
\end{tabular}

is kept at its lowest points with S1 and S4, whereas S2 and $\mathrm{S} 3$ result in an increase of 3-5\% compared to S4 because with these strategies $M G A$ interacts less with the upstream network. Therefore, MGA does not contribute to reduce the peak consumption of neighboring areas in the grid. Considering the low peak as well as the reduced cost for the DSO it is clear that the best strategy is S1, which is optimal for both MGs. However, with additional MGs it is possible that the need for coordinated MG operation will increase.

\section{B. Distribution System Operation}

After the day-ahead simulations power flow calculations were performed to validate that the uncoordinated energy scheduling solutions satisfy the network constraints (voltage limits, feeder constraints). All the simulations resulted in operation within technical limits. It should be noted, though, that the modeled network has sufficient feeder capacity and relatively low PV penetration level (14\%). A minimum interaction between the MGs and the DSO could be applied (e.g., the DSO could approve or reject a MG schedule) to ensure that the MG operation does not undermine the operation of a distribution system.

\section{Cycle-based Battery Degradation}

The link between the BESS scheduling of each strategy and the expected battery lifetime was investigated using the piecewise linear relationship of cycle life and depth-of-discharge (DoD) given in [18]. The rainflow algorithm [19], which is an electrochemically accurate model, was used to calculate the number of battery half-cycles, the cycle range and an average SOC. From these, the number of full cycles was calculated and an average $\operatorname{DoD}(D o D=1-S O C)$ was estimated.

The results showed that the proposed strategies are unsuitable for batteries with small cycle life (e.g., lead acid batteries), since the battery capacity could not be guaranteed for more than 5 years. They also showed that energy arbitrage and cost minimization, whether performed locally (S1) or for the whole system (S4), increase the number of cycles resulting in faster battery degradation. Therefore, these strategies should only be considered with high cycle life batteries (e.g., Li-ion or $\mathrm{NaS}$ batteries). Table IV summarizes the results and presents the estimated expected lifetime for Li-ion batteries.

\section{CONCLUSIONS}

This paper presents an EMS for grid-connected MGs with BESS. The proposed models evaluate day-ahead energy scheduling strategies in uncoordinated and coordinated schemes. A multi-period case study was performed on a real distribution network with two MGs. It is shown that even without coordination multiple MG-EMS that aim to minimize their individual cost also reduce the cost of the DSO. The effect on the battery lifetime was also assessed indicating that MG-EMS strategies that apply cost minimization should be combined with high cycle life batteries.

\section{ACKNOWLEDGMENT}

The authors acknowledge the contribution of Per Löveryd from Akademiska Hus in providing the data for the case study.

\section{REFERENCES}

[1] N. Hatziargyriou, "The microgrids concept," in Microgrids: architectures and control. Wiley-IEEE press, 2014, ch. 1, pp. 1-24.

[2] D. E. Olivares et al., "Trends in microgrid control," IEEE Trans. on smart grid, vol. 5, no. 4, pp. 1905-1919, July 2014.

[3] A. Ali et al., "Overview of current microgrid policies, incentives and barriers in the European Union, United States and China," Sustainability, vol. 9, no. 7, June 2017.

[4] W. Su, J. Wang, and J. Roh, "Stochastic energy scheduling in microgrids with intermittent renewable energy resources," IEEE Trans. on Smart Grid, vol. 5, no. 4, pp. 1876-1883, July 2014.

[5] M. El-Hendawi, H. Gabbar, G. El-Saady, and E.-N. Ibrahim, "Control and EMS of a grid-connected microgrid with economical analysis," Energies, vol. 11, no. 1, p. 129, Jan. 2018.

[6] B. Zhao et al., "Energy management of multiple microgrids based on a system of systems architecture," IEEE Trans. on Power Syst., vol. 33 no. 6, pp. 6410-6421, Nov. 2018.

[7] P. Tian, X. Xiao, K. Wang, and R. Ding, "A hierarchical energy management system based on hierarchical optimization for microgrid community economic operation," IEEE Trans. on Smart Grid, vol. 7, no. 5, pp. 2230-2241, Sep. 2016.

[8] S. Wang, H. Gangammanavar, S. D. Ekşioğlu, and S. J. Mason, "Stochastic optimization for energy management in power systems with multiple microgrids," IEEE Trans. on Smart Grid, vol. 10, no. 1, pp. 1068-1079, Jan. 2019.

[9] m2M-GRID, "From micro to Mega-GRID: Interactions of micro-grids in active distribution networks." [Online]. Available: https://m2m-grid.eu/

[10] M. Göransson, N. Larsson, D. Steen, and L. A. Tuan, "Cost-benefit analysis of battery storage investment for microgrid of Chalmers university campus using $\mu$-opf framework," in Proc. IEEE PowerTech, Manchester, UK, June 2017.

[11] G. C. Lazaroiu et al., "Energy-informatic-centric smart campus," in Proc. IEEE 16th Int. Conf. on Environment and Elect. Eng. (EEEIC), Florence, Italy, June 2016.

[12] M. Quashie, F. Bouffard, C. Marnay, R. Jassim, and G. Joós, "On bilevel planning of advanced microgrids,' Int. J. of Elect. Power \& Energy Syst, vol. 96, pp. 422-431, Mar. 2018.

[13] M. Farivar and S. H. Low, "Branch flow model: Relaxations and convexification-Part I," IEEE Trans. on Power Syst., vol. 28, no. 3, pp. 2554-2564, Apr. 2013.

[14] Z. Norwood, E. Nyholm, T. Otanicar, and F. Johnsson, "A geospatial comparison of distributed solar heat and power in Europe and the US," PLoS ONE, vol. 9, no. 12, p. e112442, Dec. 2014.

[15] Nord Pool. [Online]. Available: https://www.nordpoolgroup.com/

[16] Göteborg Energi. [Online]. Available: https://www.goteborgenergi.se/

[17] GAMS. [Online]. Available: https://www.gams.com/

[18] I. Alsaidan, A. Khodaei, and W. Gao, "A comprehensive battery energy storage optimal sizing model for microgrid applications," IEEE Trans. on Power Syst., vol. 33, no. 4, pp. 3968-3980, July 2018.

[19] Y. Shi, B. Xu, Y. Tan, and B. Zhang, "A convex cycle-based degradation model for battery energy storage planning and operation," in Proc. Annual American Control Conference (ACC), Wisconsin, US, Aug. 2018, pp. 4590-4596. 